\title{
Vaccine testing of a recombinant activation-associated secreted protein (ASP1) from Ostertagia ostertagi
}

\author{
P. GELDHOF, Y. MEYVIS, J. VERCRUYSSE \& E. CLAEREBOUT
}

Laboratory of Parasitology, Faculty of Veterinary Medicine, Ghent University, Salisburylaan, Merelbeke, Belgium

\section{SUMMARY}

Previous vaccination trials against the economically important cattle parasite Ostertagia ostertagi have indicated the protective capacity of activation-associated secreted proteins (ASPS). The further development of these antigens into a commercial vaccine will require their recombinant expression. The aim of the current study was to clone and express Oo-aspl in a baculovirus expression system and to evaluate the protective capacity of the recombinant protein against an $\mathrm{O}$. ostertagi challenge infection in cattle. The full coding sequence of Oo-asp1 was cloned in a baculovirus expression vector in frame with a carboxy-terminal Histidine tag and recombinant virus was used to infect an insect cell culture. Western blot analysis with anti-His and anti-Oo-ASP1 antibodies showed the production of recombinant Oo-ASP1. The cell pellet containing the recombinant was subsequently used to immunize seven calves three times intramuscularly with QuilA as adjuvant. Control animals were solely injected with the QuilA adjuvant. The challenge infection with $\mathrm{O}$. ostertagi consisted of 30000 L3 larvae per animal given over 30 days (1000 larvaelday, 5 days/week) and started the same day as the final immunization. Immunization with the recombinant Oo-ASP1 did not result in any level of protection against the challenge infection. There was no reduction in faecal egg output or in worm burdens. Moreover, Western blot analyses and ELISA indicated that, although the animals raised an antibody response against the recombinant $O o-A S P 1$, there was hardly a response against the native Oo-ASP1, suggesting that the baculovirus expressed

Correspondence: Peter Geldhof, Laboratory of Parasitology, Faculty of Veterinary Medicine, Ghent University, Salisburylaan 133, B-9820 Merelbeke, Belgium (e-mail: peter.geldhof@ugent.be). Received: 4 October 2007

Accepted for publication: 30 October 2007 recombinant was wrongly folded or lacked essential secondary modifications. Further analysis of the structure of the native ASPs and their glycosylations is being done.

Keywords ASP, Ostertagia ostertagi, recombinant, vaccination

\section{RESEARCH NOTE}

Previous vaccination trials against the economically important cattle parasite Ostertagia ostertagi have demonstrated the protective capacity of a protein fraction termed ES-thiol (1-3). This antigen fraction is purified from adult excretorysecretory material and is highly enriched for two activationassociated secreted proteins (ASPs), Oo-ASP1 and OoASP2 (4), and cysteine protease activity (1-3). Intramuscular immunization in combination with QuilA as adjuvant consistently resulted in a significant reduction in cumulative faecal egg counts of around $60 \%$. This reduction lasted for about 2 months after the first infection with L3 larvae. In a recent study, Meyvis et al. (3) demonstrated the protective capacity of a purified ASP containing fraction. The ES-thiol fraction was subfractionated by Q-Sepharose anion exchange chromatography to separate the ASPs from the cysteine proteases and other proteins. Three subfractions were obtained, an 'ASP-enriched', a 'cysteine protease-enriched' and a 'rest' fraction. SDS-PAGE analysis, enzyme assays and Western blotting indicated that the ASP-enriched fraction was virtually pure ASPs and free of any cysteine proteinase activity. Vaccination with this fraction resulted in a $74 \%$ reduction in cumulative faecal egg counts and significantly smaller adult male and female worms. It can be anticipated that this level of protection would be sufficient for a commercial vaccine (5). It would protect calves against gastroenteritis during their first grazing season and allow them to develop a natural immunity without production loss. Because it is very difficult to obtain large quantities of 
these native ASPs, further development of these antigens into a commercial vaccine will therefore rely on their recombinant expression.

The aim of the current study was to clone and express Oo-asp1 in a baculovirus expression system and to evaluate the protective capacity of the recombinant protein against an $O$. ostertagi challenge infection in cattle.

The full coding sequence of Oo-aspl (4) was cloned in the baculovirus expression vector $\mathrm{pVec} 35$ (Intervet International B.V. Boxmeer, The Netherlands) in frame with a carboxyterminal Histidine tag. The recombinant baculovirus was constructed by co-transfection of the Oo-asp $1 \mathrm{pVec} 35$ vector and a wild-type virus into $S f g$ insect cells to promote in vivo recombination. This was essentially done as previously described by Schijns et al. (6). A recombinant virus was isolated, purified and amplified for subsequent infection of an insect cell culture in order to produce the Oo-ASP1 recombinant antigen. Cell supernatant and cell pellet were inactivated with $25 \mathrm{kGray} \gamma$-irradiation and run on SDSPAGE gels. A Coomassie stained gel of the supernatant and cell pellet is shown in Figure 1, panel a (lanes 1 and 2). This material was tested for antigen production by Western blotting with conjugated anti-His antibodies and monospecific anti-Oo-ASP1 antibodies (4). The results of these blots are shown in Figure 1, panel a. One band in the cell supernatant and three to four bands in the cell pellet, all around $25 \mathrm{kDa}$ were detected using an anti-His antibody (lanes 3 and 4). A similar pattern was visible using monospecific antibodies against Oo-ASP1 (lanes 5 and 6).

The vaccination trial with the recombinant Oo-ASP1 was essentially done as previously described in Geldhof et al. (1). Fourteen male 7-months-old calves were randomized over two groups of seven animals. The animals were immunized three times by intramuscular injection in the neck at 3-week intervals. One group was administered $100 \mu \mathrm{g}$ of the Oo-ASP1 containing cell pellet in combination with $750 \mu \mathrm{g}$ QuilA adjuvant (Superfos Biosector). The control animals were solely injected with the QuilA adjuvant. The challenge infection with $O$. ostertagi consisted of $30000 \mathrm{~L} 3$ larvae per animal given over 30 days (1000 larvae/day, 5 days/week) and started the same day as the final immunization. The animals were observed daily for adverse reactions to the immunizations and for clinical signs of ostertagiosis. The animals were bled 1 week after each immunization and at the time of necropsy 24 days after the final challenge infection. Faecal egg counts were done three times a week from 20 days after the first challenge infection until necropsy. The faecal egg output was determined using a modified McMaster technique with a sensitivity of 25 eggs per gram (EPG). The cumulative faecal egg output was calculated for each animal as described by Vercruysse et al. (7). At necropsy, the abomasal washings and the abomasal digests were performed as previously (a)

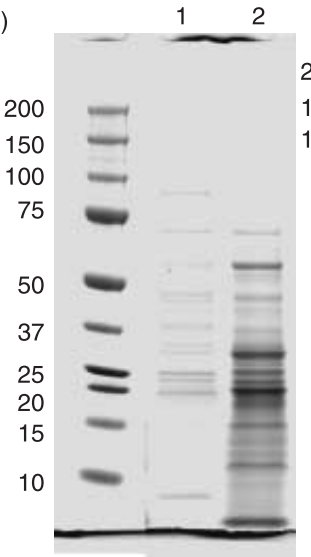

3
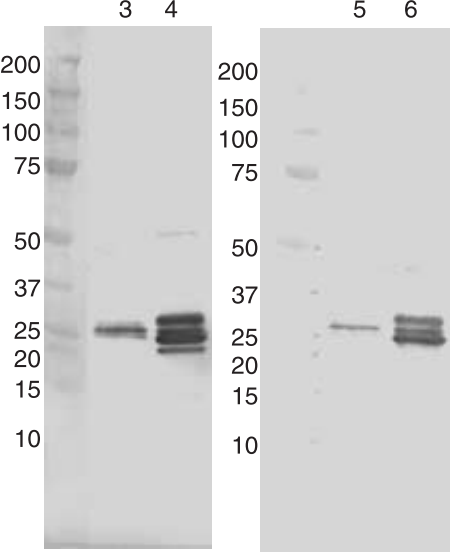

(b)

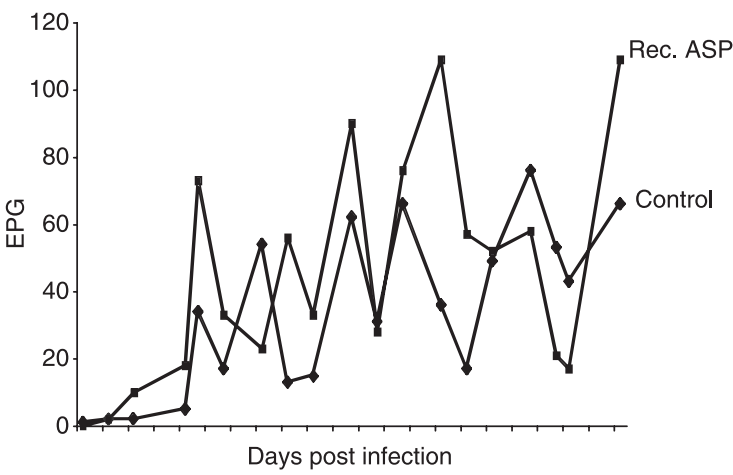

(c)

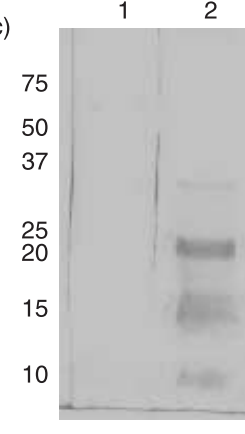

(d) 2.200

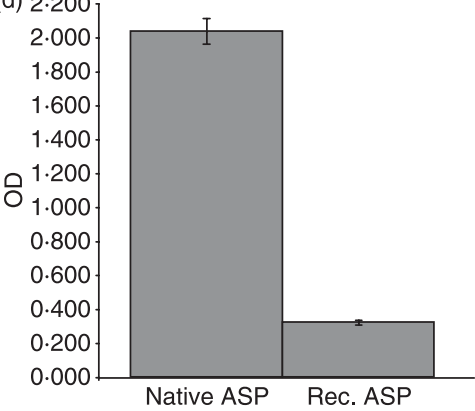

Figure 1 (a) Protein profile and Western blots of the insect cell supernatant and pellet. Lanes 1 and 2: Coomassie stained gel of cell supernatant and cell pellet, respectively. Lanes 3 and 4: Western blot of cell supernatant and cell pellet developed with anti-His antibody. Lanes 5 and 6: Western blot of cell supernatant and cell pellet developed with anti-Oo-ASP1 antibody, produced as described by Geldhof et al. (2003) (4). (b) Geometric mean of the faecal egg output during the 2-month period of the vaccination trial of the animals vaccinated with QuilA (control) and the animals vaccinated with the recombinant Oo-ASP1 (rec. ASP). (c) Serum antibody response of the animals vaccinated with the recombinant Oo-ASP1 against the cell pellet. Lane 1: pre-immune serum, lane 2 serum taken 1 week after the second immunization. (d) Antibody response in animals vaccinated with the recombinant Oo-ASP1 against the native Oo-ASP1. A native 'ASP-enriched' fraction was used to coat the ELISA plate. Serum from the animals vaccinated with the recombinant Oo-ASP1 was compared with the response from animals vaccinated with the native 'ASP-enriched' fraction. 
Table 1 Numbers of animals per group (n), cumulative faecal egg counts $($ FEC + range), geometric mean total worms counts (+ range) and percentage L4 stage

\begin{tabular}{lllll}
\hline Group & $n$ & FEC & No. of worms & $\%$ L4 \\
\hline Control & 7 & $2986(1288-7263)$ & $5771(2900-8350)$ & $0 \cdot 3$ \\
Rec. ASP1 & 7 & $3173(1438-5950)$ & $7158(1700-10050)$ & 0 \\
\hline
\end{tabular}

described (1). Two percent of both the abomasal washings and digests were analysed to determine the worm burden. Geometric mean egg and worm counts were calculated after transformation of the individual counts to $\ln (\operatorname{count}+1)$. The significance of differences in the parasitological parameters (cumulative faecal egg counts, worms counts) between the two groups was investigated by a one-tailed Mann-Whitney $U$-test.

The results of the faecal egg counts during the course of the vaccination trial are shown in Figure 1, panel b as geometric means. During most of the vaccination trial the geometric mean egg counts for the rOo-ASP1 vaccinated animals were higher compared to the control animals. The parasitological data are summarized in Table 1. There was no significant difference in the number of adult worms between the two groups.

The serum antibody responses of the calves to the immunizations were evaluated by Western blotting using sera collected 1 week after the second immunization. Ten micrograms of the recombinant cell pellet was fractionated on $10 \%$ SDS-PAGE gels under reducing conditions and subsequently blot transferred to PVDF membranes. After overnight blocking in $10 \%$ horse serum in PBS Tween 20 (PBST), the blots were probed for $2 \mathrm{~h}$ with pooled sera (diluted $1: 400$ in $2 \%$ horse serum PBST), followed by addition of the conjugate (rabbit antibovine HRPO, Sigma) at a $1: 8000$ dilution in $2 \%$ horse serum PBST. The immunoreactive antigens were visualized by addition of $0.05 \% 3,3$ diaminobenzidine tetrachloride in PBS containing $0 \cdot 01 \% \mathrm{H}_{2} \mathrm{O}_{2}(\mathrm{v} / \mathrm{v})$. The results are shown in Figure 1, panel c. No antibodies to the antigens were detected in the pre-immune serum (lane 1), while serum taken after the immunization strongly recognized different antigens in the cell pellet, especially a region around $25 \mathrm{kDa}$, coinciding with the size of the rOo-ASP1 (lane 2). There was no reactivity from serum of the control group (results not shown).

An ELISA was used to measure the presence of crossreactive antibodies against the native 'ASP-enriched' fraction in the animals vaccinated with the baculovirus expressed Oo-ASP1. The 'ASP-enriched' antigen fraction was purified as previously described in Meyvis et al. (3) and coated on the ELISA plate at $5 \mu \mathrm{g} / \mathrm{mL}$ with $150 \mu \mathrm{L} / \mathrm{well}$ overnight at $4^{\circ} \mathrm{C}$. After three washes with PBST, the plate was blocked with $200 \mu \mathrm{L} /$ well of $2 \%$ horse serum PBST for $2 \mathrm{~h}$ at room temperature. The primary antibody, that is, pooled serum from the vaccinated animals, was added at a dilution of $1: 500$ in $2 \%$ horse serum PBST. After $2 \mathrm{~h}$ at $37^{\circ} \mathrm{C}$, the conjugate was added (rabbit antibovine HPRO, Sigma) at a dilution of $1: 5000$ in $2 \%$ horse serum PBST and the plate incubated for $2 \mathrm{~h}$ at $37^{\circ} \mathrm{C}$. $o$-Phenylenediamine $0.1 \%$ in citrate buffer $(\mathrm{pH} 5.0)$ served as substrate and optical density (OD) was measured at $492 \mathrm{~nm}$. The OD of the baculo Oo-ASP1 vaccinated animals was compared with the antibody response in animals vaccinated with the native ASP-enriched fraction from a previous vaccination trial (3). The results of the ELISA are shown in Figure 1, panel d. The rOo-ASP1 vaccinated animals showed an OD value of $0 \cdot 321$, which is only $15 \%$ of the OD value of animals vaccinated with the native antigens (OD 2.038).

The data presented here indicate that the recombinant version of Oo-ASP1 tested in this study was unable to induce any protection. Moreover, immunization with the recombinant antigen hardly resulted in the production of cross-reactive antibodies against the native version of Oo-ASP1. A possible explanation is that the relative quantities of ASP1 in the insect cell pellet might have been too low to induce a proper cross-reactive antibody response. In the vaccine trial described by Meyvis et al. (3) animals were vaccinated with approximately $30 \mu \mathrm{g}$ of the native 'ASP-enriched' fraction. In the current experiment, a $100-\mu \mathrm{g}$ of the antigen mixture was injected, a large portion of which are the ASP1 bands. In addition, the Western blot analysis indicated that the immunized animals strongly recognized the recombinant ASP1 bands. It is therefore questionable if vaccinating with a higher dose would increase the titre of cross-reactive antibodies and subsequently induce protection. Alternatively, the baculovirus expressed recombinant could be wrongly folded or lack essential secondary modifications and might therefore not induce cross-reactive antibodies. The baculovirus expression system has previously been successfully used to express host-protective antigens from Fasciola hepatica (8), Schistosoma japonicum (9) and Ancylostoma caninum (10). However, it was never used to express a nematode ASP molecule. All previously tested ASP recombinants were bacterial and/or yeast expressed versions. This was the case for ASPs from hookworms (11-14), Haemonchus contortus (15) and Onchocerca volvulus (16). In the case of the $O$. ostertagi ASP, a bacterially expressed Oo-ASP1 previously proved to be insoluble and could not be refolded or solubilized (Y. Meyvis, unpublished results), most likely due to the high cysteine content in this type of molecules (4). A further analysis of the structure of the native ASPs and their glycosylations is being done. It will investigate the peptide and/or glycan epitopes that are important for the protection. On the other hand, it is important to note that the native 
ASP fraction consisted of two ASPs, that is, ASP1 and ASP2. Although ASP1 is far more abundant in the protective fraction and shares similarity with ASP2, it may very well be that ASP2 or a mix of both molecules is required for the protection.

\section{ACKNOWLEDGEMENTS}

P.G. is a recipient of Marie Curie EU reintegration grant (No. 028870) and is a Postdoctoral Fellow of the Fund for Scientific Research - Flanders (Belgium) (F.W.O. - Vlaanderen). Y.M. is supported by a grant from Ghent University (BOF 01109405). This work was partly financed by Intervet International B.V. We thank V. De Maere, I. Vercauteren and $\mathrm{S}$. Casaert for their assistance.

\section{REFERENCES}

1 Geldhof P, Claerebout E, Knox D, Vercauteren I, Looszova A \& Vercruysse J. Vaccination of calves against Ostertagia ostertagi with cysteine proteinase enriched protein fractions. Parasite Immunol 2002; 24: 263-270.

2 Geldhof P, Vercauteren I, Vercruysse J, Knox DP, van den Broeck W \& Claerebout E. Validation of the protective Ostertagia ostertagi ES-thiol antigens with different adjuvantia. Parasite Immunol 2004; 26: 37-43.

3 Meyvis Y, Geldhof P, Gevaert K, Timmerman E, Vercruysse J \& Claerebout E. Vaccination against Ostertagia ostertagi with subfractions of the protective ES-thiol fraction. Vet Parasitol 2007; 149: 239-245.

4 Geldhof P, Vercauteren I, Gevaert K, et al. Activation-associated secreted proteins are the most abundant antigens in a host protective fraction from Ostertagia ostertagi. Mol Biochem Parasitol 2003; 128: 111-114.

5 Claerebout E, Knox DP \& Vercruysse J. Current research and future prospects in the development of vaccines against gastrointestinal nematodes in cattle. Expert Rev Vaccines 2003; 2: $147-157$.

6 Schijns VE, Scholtes NC, Zuilekom HI, Sanders LE, Nicolson L \& Argyle DJ. Facilitation of antibody forming responses to viral vaccine antigens in young cats by recombinant baculovirusexpressed feline IFN- $\gamma$. Vaccine 2002; 20: 1718-1724.

7 Vercruysse J, Dorny P, Hong C, et al. Efficacy of doramectin in the prevention of gastrointestinal nematode infections in grazing cattle. Vet Parasitol 1993; 49: 51-59.

8 Hillyer GV. Fasciola antigens as vaccines against fasciolosis and schistosomiasis. J Helminthol 2005; 79: 241-247.

9 Wu DZ, Lu ZY \& Yu XB. Development of a vaccine against Schistosoma japonicum in China: a review. Acta Tropica 2005; 96: $106-116$.

10 Bungiro R \& Cappello M. Hookworm infection: new developments and prospects for control. Curr Opin Infect Dis 2004; 17: 421426.

11 Ghosh K \& Hotez PJ. Reductions in mouse hookworm burden after vaccination with Ancylostoma caninum secreted protein 1. J Infect Dis 1999; 180: 1674-1681.

12 Sen L, Ghosh K, Bin Z, et al. Hookworm burden reductions in $\mathrm{BALB} / \mathrm{c}$ mice vaccinated with recombinant Ancylostoma secreted proteins (ASPs) from Ancylostoma duodenale, Ancylostoma caninum and Necator americanus. Vaccine 2000; 18: 1096-1102.

13 Mendez S, Zhan B, Goud G, et al. Effect of combining the larval antigens Ancylostoma secreted protein 2 (ASP-2) and metalloprotease 1 (MTP-1) in protecting hamsters against hookworm infection and disease caused by Ancylostoma ceylanicum. Vaccine 2005; 23: 3123-3130.

14 Goud GN, Zhan B, Ghosh K, et al. Cloning, yeast expression, isolation and vaccine testing of recombinant Ancylostomasecreted protein (ASP) -1 and ASP-2 from Ancylostoma ceylanicam. J Infect Dis 2004; 189: 919-929.

15 Vervelde L, Van Leeuwen MAW, Kruidenier M, et al. Protection studies with recombinant excretory/secretory proteins of Haemonchus contortus. Parasite Immunol 2002; 24: 189-201.

16 MacDonald AJ, Tawe W, Leon A, et al. Ov-ASP1, the Onchocerca volvulus homologue of the activation associated secreted protein family is immunostimulatory and can induce protective anti-larval immunity. Parasite Immunol 2004; 26: 53-62. 